\title{
Breve mirada \\ al contexto educativo internacional y la necesidad de la participación docente en un ciclo de mejora continua
}

Departamento de Formación y Actualización Académica

El universo se parece cada vez menos a una gran maquinaria y cada vez más a un gran pensamiento.

Sir James Jeans

\section{La importancia de la formación docente ante la nueva dinámica mundial}

$\mathrm{E}$

1 contexto internacional actual nos confronta con una sinergia global, contenida en una red de complejas interacciones, que afecta las predicciones de futuro en todos los ámbitos, incluyendo el educativo. Ello nos obliga a flexibilizarnos como individuos y como organizaciones a cualquier nivel, para lograr una adaptación adecuada con el entorno local, nacional e internacional (Gershenson, 2016).

Ante este panorama, toma relevancia el papel de los docentes, actores principales de la educación (junto con los estudiantes) (UAA, 2007), de quienes se espera una adaptación más inmediata, flexible y creativa, entre otros rasgos, que les permita contribuir desde los espacios educativos, en la construcción de soluciones y alternativas equitativas, incluyentes y sustentables, con las cuales se aborden las diversas problemáticas presentes en el entorno. En este sentido, Roeser (2010) afirma: "Se requiere un cambio radical; replantear la función de los profesores ante el nuevo orden político, social e intercultural". Lo anterior, haciendo partícipe activo al estudiante, para contribuir de esta manera a una educación centrada en su aprendizaje, y orientarle hacia la búsqueda de una mayor conciencia sobre su forma de aprender, al desarrollo de actitudes, valores, habilidades y conocimientos, que le permitan transitar por un camino de aprendizaje continuo durante toda su vida.

Por ello, la formación de los docentes se considera elemental para lograr los fines educativos, misma que contempla, tanto competencias pedagógicas sólidas, como un conjunto de conocimientos (Roeser, 2010): "sobre las necesidades de los alumnos, de las materias a impartir" (área de especialización del docente) y "sobre la gestión de las emociones (medios para diseñar modelos de comportamiento responsable)", los cuales de forma integrada pueden dar como resultado "profesores eficaces".

\section{Algunos antecedentes y objetivos para la educación a nivel internacional}

De acuerdo con lo anterior, desde el ámbito internacional se proponen recomendaciones y se realizan acciones de forma consensuada, para apoyar la adaptación de los sistemas educativos a la dinámica mundial. La Organización de las Naciones Unidas para la Educación, la Ciencia y la Cultura (UNESCO), en colaboración con otras instituciones 
internacionales: Fondo de las Naciones Unidas para la Infancia (UNICEF), Fondo de Población de las Naciones Unidas (UNFAP), Organización de las Naciones Unidas Mujeres (ONU MUJERES) y Alto Comisionado de las Naciones Unidas para los Refugiados (ACNUR) organizó el Foro Mundial sobre la Educación 2015, en Incheon, República de Corea, en el cual fue aprobada "la Declaración de Incheon para la Educación 2030, en la que se presenta una nueva visión de la educación para los próximos 15 años" (UNESCO, 2015: 5). Con la participación de mil 600 asistentes de 160 países, el foro permitió el planteamiento de nuevos retos y la reflexión sobre los avances logrados respecto a los objetivos propuestos en otros encuentros celebrados y que anteceden a este evento, (ver Tabla 1).

A partir de la dinámica mundial derivada de los nuevos órdenes y el surgimiento de otros retos, principalmente en torno a fomentar la sustentabilidad por medio de la educación, se establece el Objetivo de Desarrollo Sostenible 4. "Garantizar una educación inclusiva y equitativa de calidad y promover oportunidades de aprendizaje permanente para todos" (UNESCO, 2015: 7):

La visión se inspira en una concepción humanista de la educación y del desarrollo basada en los derechos humanos y la dignidad, la justicia social, la inclusión, la protección, la diversidad cultural, lingüística y étnica, y la responsabilidad y la rendición de cuentas compartidas. Reafirmamos que la educación es un bien público, un derecho humano fundamental y la base para garantizar la realización de otros derechos. Es esencial para la paz, la tolerancia, la realización humana y el desarrollo sostenible. Reconocemos que la educación es clave para lograr el pleno empleo y la erradicación de la pobreza. Centraremos nuestros esfuerzos en el acceso, la equidad, la inclusión, la calidad y los resultados del aprendizaje, dentro de un enfoque del aprendizaje a lo largo de toda la vida.

\section{Año Ciudad Evento}

1990 Jomtien Se propuso el objetivo "Educación para Todos".

2000 Dakar Se reitera el objetivo "Educación para Todos".

2014 Mascate Acuerdo de Mascate.

Reunión Mundial sobre la Educación para Todos (ЕРT). Se conforman las bases para las metas de educación propuestas por el Grupo de Trabajo Abierto sobre los Objetivos de Desarrollo Sostenible (ODS).

2015 Incheon Educación 2030. Declaración de Incheon. Se establece el oDs 4:

"Garantizar una educación inclusiva y equitativa de calidad y promover oportunidades de aprendizaje permanente para todos".

Tabla 1. Principales encuentros mundiales para el consenso del rumbo de la educación, a partir de Educación 2030. Declaración de Incheon (UNESCO, 2015).

\section{Orientaciones institucionales para la formación docente}

Acorde con esas aspiraciones educativas en el contexto mundial prevaleciente, la Universidad Autónoma de Aguascalientes (UAA), a través de su Modelo Educativo Institucional (MEI), promueve en los docentes el desarrollo de rasgos y tareas orientadas al desarrollo equilibrado de su ser, con un enfoque humanista, que les permita asumir un compromiso responsable con la docencia que ejercen para mejorar el aprendizaje de los estudiantes.

En este sentido, la educación en la UAA "se concibe como un proceso intencional y sistemático cuya finalidad es la formación integral de la persona" (UAA, 2007: 6), identificada por una calidad equitativa, pertinente, permanente, responsable, innovadora, flexible, factible, sistémica, altamente especializada y enfocada al estudiante y su aprendizaje; asimismo, por una formación humanista caracterizada por los atributos de la formación profesional, intelectual, actitudinal, valoral, fisica, cultural y social. De acuerdo con lo anterior, el MEI es una guía que provee a los docentes de una rica conceptualización sobre la educación que se requiere ejercer en la UAA, su filosofía y los ideales a los cuales se aspira llegar. 
Para lograr los preceptos del MEI es necesaria la participación de la comunidad docente y también el apoyo del personal administrativo de la institución; a través de una estructura departamental con 65 unidades de naturaleza académica y 39 administrativas, distribuidas en 10 centros académicos y siete direcciones generales, respectivamente, la UAA integra una amplia variedad de disciplinas del conocimiento y de gestión, que le permiten el establecimiento de proyectos institucionales de forma colegiada e interdisciplinaria para su adaptación a un mundo, cuya constante es el cambio; entre éstos, uno de los más relevantes para el quehacer universitario es el Plan de Desarrollo Institucional 20162024 (PDI), fundamentado en cuatro ejes principales: Docencia, Investigación, Vinculación y Gestión, destacando el primero, por referirse directamente a la labor docente en la institución (UAA, 2016: 88):

Formar integralmente y con un alto sentido humanista a los estudiantes en programas y procesos educativos de alta calidad reconocidos nacional e internacionalmente; de esta forma, los egresados son altamente competentes y contribuyen de forma efectiva al desarrollo sustentable de su entorno.

A partir de lo anterior, el PDI plantea cuatro objetivos entre los que se puntualizan dos por vincularse más directamente con el fortalecimiento del personal académico. El número dos: "Contar con una plantilla de profesores habilitados con posgrado y actualizados en metodologías de enseñanza, tecnologías educativas innovadoras y en un segundo idioma de acuerdo al MEI, para incrementar la calidad educativa" (UAA, 2016: 90); y el objetivo número tres que indica la necesidad de "Fortalecer el trabajo colegiado intradepartamental e interdepartamental para consolidar la calidad educativa" (ídem).

\section{Estrategias para la formación en la UAA}

Para contribuir al logro de los objetivos referidos, el Departamento de Formación y Actualización Académica (DEFAA), a través del Programa Institucional de Formación y Actualización Docente (PIFOD), promueve el desarrollo integral de los profesores en ocho áreas: "Identidad institucional,

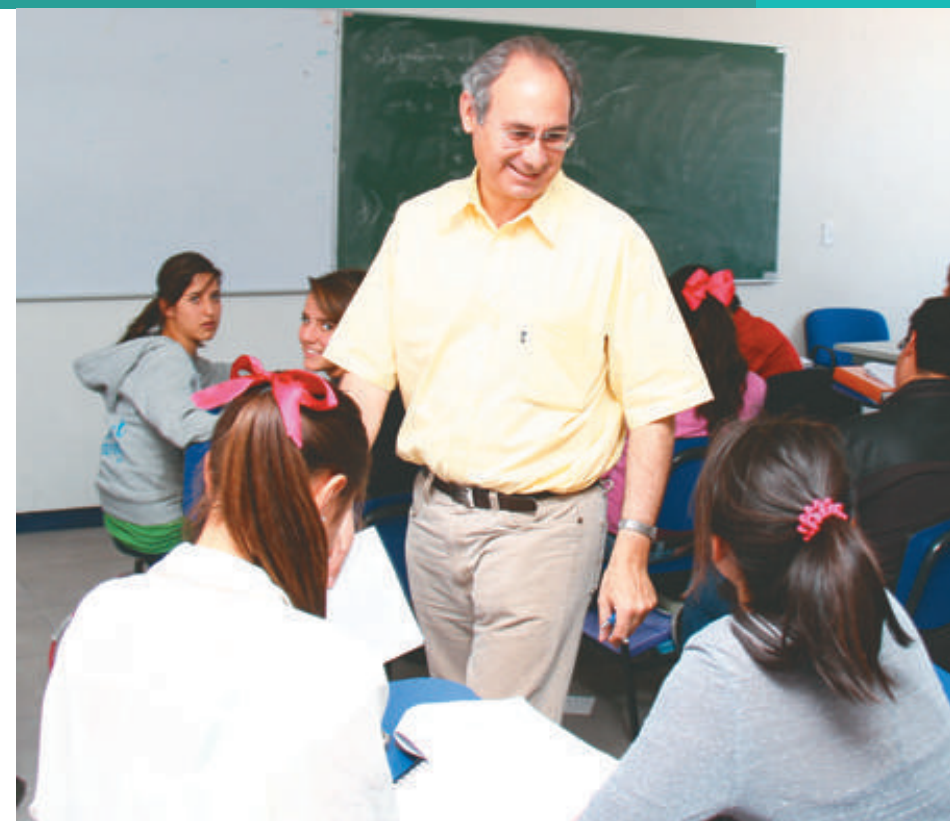

Diseño curricular, Metodologías de enseñanza, Recursos didácticos y TIC aplicadas a la educación, Evaluación educativa, Formación humanista, Lenguas extranjeras y Tutoría" (UAA, 2013: 58-59).

Por ello, el DEFAA desarrolla diversas estrategias como son la oferta de cursos generales y especiales en cuatro periodos al año; la edición de la revista semestral DOCERE, a la fecha con 16 números publicados; la producción semanal del programa de radio "El Gis", con 23 años de trayectoria; la edición semanal del boletín informativo "Formación Docente", distribuido a los mil 231 docentes suscritos a la REDOCENTE ${ }^{1}$; así como la publicación para los mil 664 seguidores en redes sociales ${ }^{2}$ sobre investigación educativa, divulgación científica, recursos didácticos y TIC, cultura, historia, frases para reflexionar, entre otros contenidos sobre el acontecer educativo para contribuir a mejorar la práctica docente.

Acorde con lo antes referido, y como estrategia principal del DEFAA, en la gráfica 1 se presenta la oferta de cursos implementada durante el año 2016, distribuida en las ocho áreas ${ }^{3}$ de formación docente:

1 Lista de distribución masiva al correo electrónico.

$2 \bigoplus$ Formación Docente uAA (DEFAA); 0 @DEFAA_UAA.

3 Algunos cursos corresponden a dos áreas, por lo cual se ubicaron en la que presentan una mayor tendencia: en Identidad institucional se incluye uno combinado con la Formación humanista y tres con el Diseño curricular; en Metodologías de enseñanza, uno con Formación humanista; y en Recursos didácticos y Tıc aplicadas a la educación, uno con Diseño curricular, cinco con Evaluación educativa y dos con Formación humanista. 


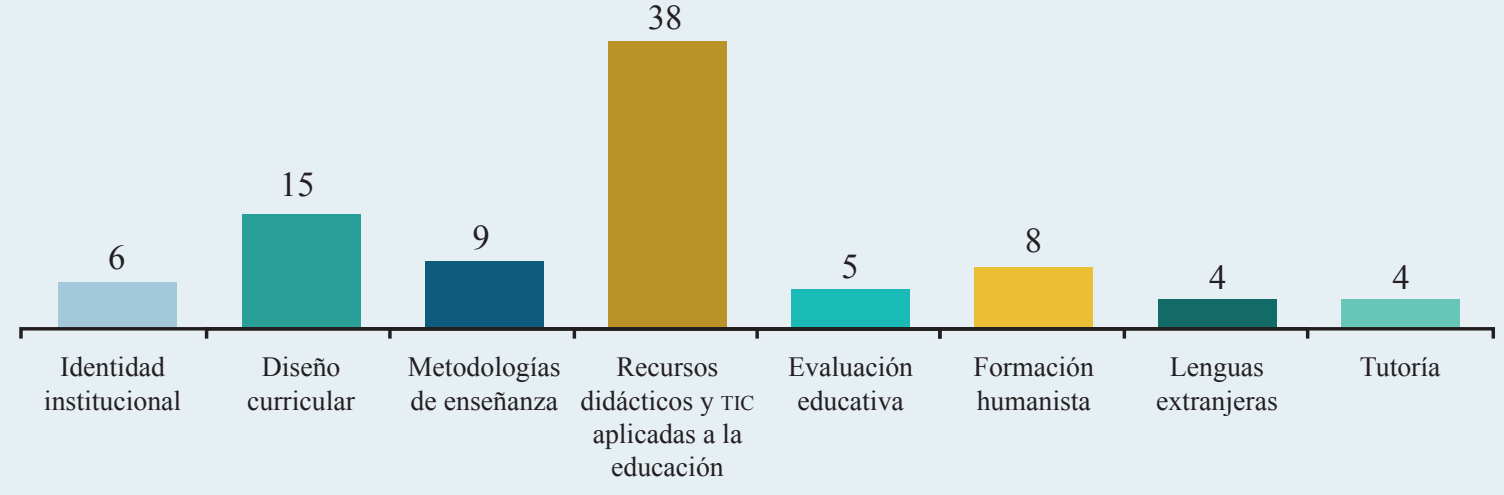

Gráfica 1. Cursos implementados por área de formación docente durante el año 2016.

Fuente: Base interna de datos del Departamento de Formación y Actualización Académica (2016).

Entre las temáticas que principalmente se abordan en los cursos impartidos son:

- Identidad institucional: inducción a la UAA, modelo educativo e historia de la educación.

- Diseño curricular: en su mayoría cursos para el diseño de experiencias de aprendizaje, rediseño de planes de estudio y diseño de programas de materia.

- Evaluación educativa: estrategias básicas para la evaluación de los aprendizajes, evaluación institucional, adaptación y uso de instrumentos de cotejo y de pruebas objetivas.

- Formación humanista: desarrollo de una cultura ambiental, la educación y la perspectiva de género en las aulas, apreciación de las artes y la cultura, desarrollo personal y la reflexión docente.

- Lenguas extranjeras: desarrollo profesional del docente de idiomas y aprendizaje del idioma inglés.

- Metodologías de enseñanza: habilidades básicas para la docencia a través de aprendizaje-servicio, por casos, por problemas y colaborativo; y la socioafectividad como método de enseñanza.

- Recursos didácticos y TIC aplicadas a la educación: manejo de recursos de la Biblioteca Digital de la UAA, manejo de recursos tecnológicos institucionales y de otros recursos digitales abiertos en los procesos de enseñanza y aprendizaje (plataforma educativa institucional "Ámbito Académico", pantallas en aula) y redacción.
- Tutoría: introducción a la tutoría, desarrollo humano del estudiante, la tutoría frente al enfoque por competencias y estrategias de atención y seguimiento académico.

Es importante referir que entre otros insumos, en el diagnóstico anual de necesidades de formación docente que se integra para implementar la oferta de cursos generales referida, se consideran los resultados de las evaluaciones de los estudiantes a los profesores, y otras recomendaciones de organismos evaluadores de programas educativos a nivel nacional e internacional (UAA, 2015).

\section{Participación del profesorado en los cursos de formación docente}

Los servicios institucionales que se ofrecen a los docentes, a través del DEFAA, están diseñados para mantener un ciclo de actualización permanente para la mejora continua de la práctica docente; por ello, se considera importante su participación y aprovechamiento. Al respecto, en la Gráfica 2 se muestra la población de docentes adscritos a los diferentes centros académicos y su participación, respectivamente, en los cursos antes referidos, durante el año 2016.

Por otro lado, los profesores pueden optar por realizar cursos en otras instituciones, posgrados en Educación, asistir a encuentros, congresos u otro tipo de eventos con el apoyo de sus centros académicos de adscripción, como alternativas para su formación docente. Lo importante es fomentar la mejora continua sobre las prácticas docentes en la institución. 
Total de personal académico — Número de profesores / participantes

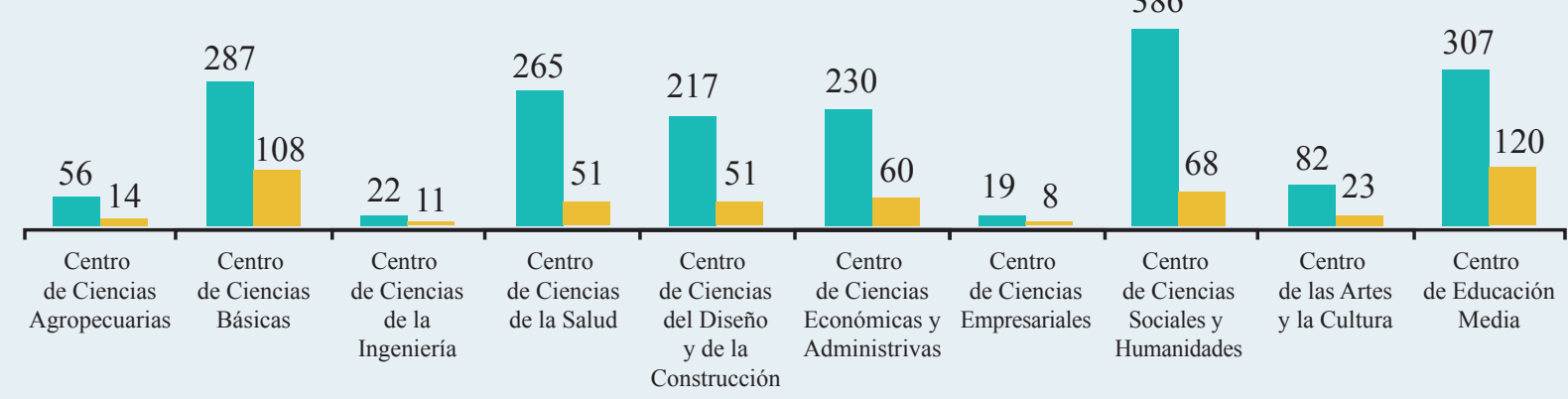

Gráfica 2. Participación en cursos de formación docente durante el año 2016.

Fuente: Base interna de datos del Departamento de Formación y Actualización Académica (2016).

\section{Conclusión}

Es complejo y largo el camino por recorrer para alcanzar las expectativas de calidad, inclusión y equidad educativa manifestadas; asimismo, es elemental contar con un amplio panorama sobre el contexto en el que se desempeña la labor educativa de las instituciones, misma que les permita adaptarse a las demandas del entorno, con el trabajo colegiado eficiente, la colaboración y la voluntad de las comunidades educativas, donde cada uno aporte con responsabilidad y agradecimiento a esta noble tarea de la educación, para alcanzar los objetivos trazados.

Sigamos avanzando hacia ese rumbo de mejora continua, por el que ha transitado la UAA desde su concepción hasta la actualidad, lo anterior ha permitido su consolidación como una institución de vanguardia y calidad reflejadas en los reconocimientos obtenidos por las distintas acreditaciones nacionales e internacionales de sus programas educativos, procesos de gestión y docentes adscritos, para que todo ello se exhiba oportunamente en la atención a las necesidades del desarrollo en el contexto local frente a una dinámica donde el cambio es la constante.

Como afirma Amina J. Mohammed (vicesecretaria general de la ONU, de origen nigeriano): "El logro de los Objetivos de Desarrollo Sostenible en todo el mundo aliviará las tensiones, mejorará la vida de mujeres y hombres y creará una base sólida para la estabilidad y la paz en todas las sociedades" (ONU, 2017).

\section{Fuentes de consulta}

Gershenson, C. (2016). Curso en línea: El Pensamiento Sistémico. Coursera. México: UNAM. Recuperado el 27 de febrero de 2017, en: http://bit.ly/2nqwvIu

ONU (2017). Centro de Noticias ONU. Recuperado el 8 de marzo de 2017, en: http://bit.ly/2rrl6ZH.

Roeser, R. (20 de junio de 2010). La revolución educativa. Entrevista de Eduart Ponset. En Redes Educativas. España: Canal A la Carta Televisión y Radio. Recuperada el 12 de marzo de 2017, en: http://bit.ly/1x126Ck

UAA (2007). Modelo Educativo Institucional. En Correo Universitario, Séptima Época, Núm. 15, publicado el 29 de mayo de 2015. Primera reimpresión. México: UAA.

UAA (2016). Diagnóstico de necesidades de formación y actualización docente para la planeación de los cursos generales 2017. México: UAA-DGDP-DEFAA (Documento mecanoescrito, no publicado)

UAA (2016). Plan de Desarrollo Institucional 2016 2024. Trazando el rumbo institucional. México: UAA.

UAA (2013). Programa Institucional de Formación y Actualización Docente. México: UAADGDP-DEFAA. Recuperado el: 8 de marzo de 2017, en: http://bit.ly/2oDwOzz

UNESCo (2015). Educación 2030. Declaración de Incheon y Marco de Acción para la realización del Objetivo de Desarrollo Sostenible 4. Paris: UNESCO. Recuperado el 2 de febrero de 2017, en: http://bit.ly/2ij2B6k 